Pola

\title{
ORNITONIMIA ISTRIANA: IL PETTIROSSO
}

0.

Il lavoro presenta un commento linguistico (soprattutto etimologico) ai nomi del pettirosso nelle parlate istriane (istroveneto, istrioto, istrorumeno, croato, sloveno e montenegrino). Abbiamo raccolto i nomi in 135 luoghi della regione istro - quarnerina (isola di Veglia compresa). Un elenco completo di tutti i nomi raccolti per questa specie verrà dato alla fine del saggio. Per non confondere il lettore le parole di tutti gli idiomi si riportano con la stessa grafia (l'alfabeto croato). L'accento viene segnato come si usa nelle rispettive parlate, tranne per le parole rumene dove si adotta il sistema croato (perché corrisponde alla realtà fonetica dell' istrorumeno).

1.

Il pettirosso (Erithacus rubecula) è "un uccelletto paffuto, 'senza collo'. Gli adulti hanno petto e fronte arancio, parti superiori bruno oliva....." (PETERSON 154) La macchia rossa sul petto distingue nettamente il nostro uccello dagli altri simili.

2.

Il sema "rosso" si rispecchia anche nel termine latino erīthacus, $-\bar{l}$, trascrizione

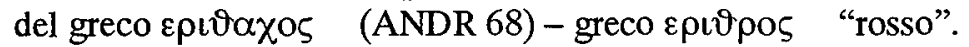

\section{1.}

Per gli idiomi istroveneti e istrioti i nomi di Erithacus rubecula sono di tipo petiròso ${ }^{1}$ - nome composto da ven. peto (< PĔCTUS, REW 6335) e roso (< RUSSUS, REW 7466). I nomi di questo tipo (alcuni derivati in -ić e -ica) nelle parlate slave della zona sono prestiti dall'istroveneto.

\section{2 .}

La maggior parte dei nomi sull'isola di Veglia corrispondono al termine ornitologico croato crvendać (SKOK I/275).

1 Lo stesso tipo prevale anche nelle parlate dell'Italia settentrionale. Cfr. ASLEF, tav. 214, carta 181; AIS III/490 e ALI 4628. 
I nomi krvāvčić (Pićan - croato) - da krv, krvav "sangue, insanguinato" (BEZLAJ II/92; SKOK II/216) + -čić; pîkeš (Valle - croato) - da pika "punto, macchia" (SKOK II/655) + -eš; e puntaruôšu (Žejane) sono isolati. L'ultimo nome è composto. Il primo elemento potrebbe essere il sostantivo rumeno punt "punto" (DLRM 680), oppure il verbo rumeno puntá "marcare con un punto" (DLRM 680), ma anche il veneto punto (< PŬNCTUM, REW 6847). Il secondo elemento è rumeno roşsu "rosso" (DLRM 726) - per l'etimologia v. sopra.

3.

I rumeni di Žejane hanno ancora un nome: puljîću de nè. Il sintagma significa "uccello della neve", perché viene con la neve (come dicono i nostri informatori). L'elemento nominale del sintagma è una forma ibrida: rum. pulj "uccello" (l'etimologia verrà data in seguito) $+-i c$ (suffisso slavo) $+-u$ (desinenza rumena per il genere maschile). La parte aggettivale che segue è composta da de + nè (rum. nea "neve", DLRM 532) < NĬX, NIVE, REW 5936.

\section{1.}

Esprime lo stesso semantismo anche il nome ciacavo snîgarićc - derivazione in -ić da ciacavo snig "neve" (SKOK III/297).

4.

L'onomasiologia popolare mette in "relazione di parentela" il pettirosso e lo scricciolo (Troglodytes troglodytes). In molti luoghi dove vengono parlati i dialetti ciacavi il nome del nostro uccello viene espresso con un sintagma di tipo aggettivo possessivo dai nomi di scricciolo + punica "suocera": trilićeva punica, trljeva punica, potrljeva punica, činčiričova punica ecc. $^{2}$

Vogliamo dimostrare che questa "parentela" nasce come frutto dell' etimologia popolare.

Per dimostrarlo bisogna spiegare le forme di tipo tašča/taščica. Queste forme sono più comuni nei dialetti sloveni che in quelli croati. Il contenuto di base di queste forme è "suocera" (croato punica). Nonostante il tipo di denominazione sia vivo nell'onomasiologia popolare, ${ }^{3}$ per questi nomi vogliamo accettare la proposta etimologi-

2 Lostesso tipo di denominazione è diffuso anche altrove: trkova punica (HIRTZ 515-Lič, Sveti Juraj, Alan, Živi Bunari, Vlaka, Jesenice in Dalmazia); krajeva pelnica (HIRTZ 213 - Vrbnik); kraljeva punica (HIRTZ 213 - Vrapče, Remete); vranjkova punica (HIRTZ 551 - Brač); banj punica (HIRTZ 7 - Poljica in Dalmazia).

3 Cfr. ugorova mati, JaFa 17.4.2. 
ca di Metka Furlan che (in litteris) presuppone per le nostre forme un protoslavo *pbtašbka "uccellino". Secondo quest' ipotesi i nomi del tipo discusso avrebbero come significato di base semplicemente "uccello, uccellino".

Secondo noi, anche i sintagmi di cui il secondo elemento è punica partano dallo stesso contenuto, "uccello". A ciò ci fa pensare la forma ciacava trljêva pûlica (Pačići, Ližnjan). Il secondo elemento del sintagma è, secondo noi, una forma ibrida (il suffisso -ica è slavo) d'origine rumena (istrorum. pulj, pul "uccello" < lat. *PULLLIUS, REW $6826^{5}$ ). Il prestito rumeno puljî̀ic "uccello" esiste ancor' oggi sull'isola di Veglia (a Skrbčići, Kornić,... - nella zona che gli abitanti chiamano "Šotovento" - abbiamo sentito la parola da molti informatori).

Per concludere, pensiamo che il secondo elemento dei sintagmi di tipo "Troglodytes troglodytes" + -ova/-eva + punica siano frutto dell'etimologia popolare che ha avvicinato la forma pulica alla forma punica, probabilmente sotto l'influsso delle forme slovene e croate di tipo tašča/taščica, di cui, come abbiamo detto, il significato originario è "suocera" (slov. tašča).

4.1.

Appartiene allo stesso gruppo anche il nome potr̈ljeva pünica (Gajana). Il primo elemento del sintagma ciacavo presenta un incrocio tra due nomi che in Istria indicano lo scricciolo: potrnčić e trlj.

\section{2.}

Abbiamo ancora un nome che mette in relazione (in questo caso non di parentela) i due uccelli. A Kostrćan il pettirosso viene chiamato tâška čamâre. Il primo elemento del sintagma, come si vedrà in seguito, è in Istria molto frequente come denominazione di pettirosso. Il secondo elemento čamare "grande, cioè quella grande" (rum. máre "grande", DLRM 479 < MAJOR, -ŌRE, REW 5247) mette questo nome in opposizione con quello dello scricciolo che a Kostrćan dicesi tuâškica čamîka - istrorum. čamika "piccola, cioè quella piccola" (rum. mic"piccolo", DLRM 497 < MĪCA “bricciola", REW 5559).

\section{3.}

Alcuni nomi del pettirosso derivano dai nomi di scricciolo. Sono le forme di tipo trljevica/trljevka.

4 Anche questo tipo di denominazione è frequente. Cfr. ptičak "regulus" (SKOK III/64) e tícka "Hirundo rustica, Delichon urbica" (Šmarje).

5 Secondo Skok * pullius > rum. puiu, puică appartiene al latino dei Balcani. 
Da menzionare anche che in alcuni posti della zona le due specie hanno la stessa denominazione: per es. a Marušići e a Rovinjsko selo il pettirosso e lo scricciolo hanno lo stesso nome, rispettivamente tâščica e trljêvova pânica.

\section{5 .}

Il nome babarûšica (Skrbčići) è composto da baba + rušica. Il secondo elemento deriva dal sema "rosso" (l'etimologia l'abbiamo spiegata sopra). Il primo elemento è lo stilema $b a b a^{6}$ che mette questo nome nella stessa categoria con quelli di tipo "suocera" (croato baba "nona")."

5.

Il nome smrêkunčić (Trget) è isolato. Deriva dal ciacavo smrekva "Juniperus sabina" (SKOK III/295). La forma è in Istria più comune per i tordi e le bigie.

6.

La forma kötlarić (Vele e Male Mune) non ci è chiara.

7.

Segue l'elenco completo dei nomi di pettirosso che abbiamo raccolto nella zona istro - quarnerina. I nomi dei luoghi sono segnati così come si chiamano oggi ufficialmente in Croazia e in Slovenia. Abbreviazioni: $\mathrm{T}$ - parlate istrovenete; $\mathrm{I}$ - parlate istriote; $\mathrm{R}$ - parlate istrorumene; $\mathrm{H}$ - parlate croate; $\mathrm{S}$ - parlate slovene; $\mathrm{C}$ - parlata montenegrina.

$\begin{array}{lll}1 & \text { PLAVJE } & \text { S: peteròś } \\ 2 & \text { ŠKOFIJE } & \text { S: petoròs, petorósić, petiróśo } \\ 3 & \text { BIVJE } & \text { T: petaròśo } \\ 4 & \text { DEKANI } & \text { S: petaròš, táščica } \\ 5 & \text { BERTOKI } & \text { T: pitaròs } \\ 6 & \text { STRUNJAN } & \text { T: petiròśo } \\ 7 & \text { POBEGI } & \text { S: petoróšo } \\ 8 & \text { PRADE } & \text { S: petiróšić } \\ 9 & \text { LUCIJA } & \text { T: petoròšo }\end{array}$

$6 \quad$ Lo stilema baba potrebbe avere lo stesso valore anche nell'ittionimia. Cfr. babaćaće "Uranoscopus scaber", JaFa 6.2.1.1.; babadlaka "Penaeus kerathurus", JaFa 38.1.; babajêgulja "Carcinus maenas", $\mathrm{JaFa}$ 38.5.2.

7 GREGORI 7 riporta babica "Erithacus rubecula". 


\begin{tabular}{|c|c|c|}
\hline 10 & KUBED & S: tàščica, peteròs \\
\hline 11 & MALIJA & S: táščica, petiróšič, peteróšo \\
\hline 12 & ŠMARJE & S: táščica \\
\hline 13 & GRAČIŠČE & S: tàščica \\
\hline 14 & MAREZIGE & S: peteróś \\
\hline 15 & KORTE & S: táškeca \\
\hline 16 & DRAGONJA & S: tàščica \\
\hline 17 & SOCERGA & S: tàščica \\
\hline 18 & BREZOVICE & S: tàščica \\
\hline 19 & SAVUDRIJA & T: petiròšo \\
\hline 20 & UMAG & T: peteròšo \\
\hline 21 & MARUŠIĆI & H: petirôšo, petarôšo \\
\hline 22 & ŠKRINJARI & $\mathrm{T}$ : petoròšo \\
\hline 23 & BUJE & T: petiròśo \\
\hline 24 & KRASICA & T: petoròšo \\
\hline 25 & PERTIĆCI & $\begin{array}{l}\text { H: tâskica } \\
\text { T: peteròśo }\end{array}$ \\
\hline 26 & GROŽNJAN & T: petoròso \\
\hline 27 & OPRTALJ & $\mathrm{T}$ : petoròso \\
\hline 28 & SVERKI & T: petiròśo \\
\hline 29 & BRTONIGLA & T: petiròśo \\
\hline 30 & VIŽINADA & T: petoròśo \\
\hline 31 & MOTOVUN & $\mathrm{T}$ : petoròšo \\
\hline & & H: trlîčeva pùnica \\
\hline 32 & BELETIĆEV BRIJEG & H: tâščica, petorôšo \\
\hline 33 & TAR & T: petiròśo, trlèvica \\
\hline 34 & LABINCI & T: petoròšo \\
\hline 35 & SOLDATICI & H: tâščica \\
\hline 36 & VIŠNJAN & T: peteròśo \\
\hline 37 & ŻBANDAJ & H: tâščica \\
\hline 38 & BADERNA & H: trlîčeva pûnica, tâšča, petirôšo \\
\hline 39 & TINJAN & H: tâščica \\
\hline 40 & LOVREČ & $\begin{array}{l}\text { T: petiròśo } \\
\text { H: trlêva pûnica }\end{array}$ \\
\hline 41 & KRINGA & H: tâščica \\
\hline 42 & FUNTANA & $\begin{array}{l}\text { T: petiròšo } \\
\text { H: petirôśo }\end{array}$ \\
\hline 43 & VRSAR & $\begin{array}{l}\text { T: petiròšo } \\
\text { H: petirôš }\end{array}$ \\
\hline 44 & GRADINA & H: petirôš \\
\hline 45 & MARIĆI & H: petorôšo, snîgarić \\
\hline 46 & KANFANAR & $\begin{array}{l}\text { T: petiròšo } \\
\text { H: činčirîčova pûnica }\end{array}$ \\
\hline 47 & ROVINJSKO SELO & H: petorôšo \\
\hline 48 & KRMED & H: trličéva pûnica \\
\hline 49 & SALAMBATI & H: trlîčeva pûnica \\
\hline
\end{tabular}




\begin{tabular}{|c|c|c|}
\hline 50 & SVETVINČENAT & $\begin{array}{l}\text { T: petoròšo } \\
\text { H: petorôšo }\end{array}$ \\
\hline 51 & PETEHI & $\mathrm{H}$ : tìljeva pùnica \\
\hline 52 & DRAGUZETI & H: tîljeva pûnica \\
\hline 53 & BARBAN & $\begin{array}{l}\text { T: petoròso } \\
\text { H: trliêva pûnica }\end{array}$ \\
\hline 54 & REŽANCI & H: trljêva pûnica \\
\hline 55 & PAČIČI & H: trljêva pûlica \\
\hline 56 & BANKOVIĆI & H: potrljêvica \\
\hline 57 & GAJANA & H: potr̂ljeva pû̀nica \\
\hline 58 & HRELJIĆI & H: trljêvka \\
\hline 59 & FILIPANA & H: trljêvka, trljêwka \\
\hline 60 & RAKALJ & $\mathrm{H}$ : tr̃ljevica \\
\hline 61 & KRNICA & T: peteròš \\
\hline 62 & KRNICA LUKA & H: trljêvica, petorôšo \\
\hline 63 & PEROJ & C: peterošić \\
\hline 64 & MARČANA & $\mathrm{H}:$ trljêva pùnica \\
\hline 65 & VELI VAREŠKI & $\mathrm{H}:$ trljêvica \\
\hline 66 & VALTURA & H: petirôšo \\
\hline 67 & PULA (VELI VRH) & $\mathrm{T}$ : petoròśo \\
\hline 68 & ŠKATARI & H: peteróśo \\
\hline 69 & POMER & H: petiròšić \\
\hline 70 & LIŽNJAN & H: trljêva pûlica, petirôšo, petorôščić \\
\hline 71 & MEDULIN & $\begin{array}{l}\text { T: petiròso } \\
\text { H: petirôšo }\end{array}$ \\
\hline 72 & PREMANTURA & H: petiròšo \\
\hline 73 & ROVINJ & I: pitorùsó \\
\hline 74 & BALE & $\begin{array}{l}\text { I: pitaróšo } \\
\text { H: trljèva pùnica, pîkeš }\end{array}$ \\
\hline 75 & VODNJAN & $\begin{array}{l}\text { I: petiròśo } \\
\mathrm{H}: \text { petirôšo }\end{array}$ \\
\hline 76 & FAŽANA & I: petiróśo \\
\hline 77 & GALIŽANA & I: petoróšo \\
\hline 78 & ŠIŠAN & I: petoróśo, petaróśo \\
\hline 79 & ŠTRPED & H: tâščica \\
\hline 80 & VIDACI & H: tâščica, peterôš \\
\hline 81 & MALI MLUN & H: tâščica \\
\hline 82 & VELI MLUN & H: tâščica \\
\hline 83 & PREMCI & H: tâščica \\
\hline 84 & ČIRITEŽ & H: tâščica \\
\hline 85 & ROČ & H: tâščica \\
\hline 86 & GRADINJE & $\begin{array}{l}\text { T: petoròšo } \\
\text { H: tâščaca, petorôšo }\end{array}$ \\
\hline 87 & PIRELIĆI & $\begin{array}{l}\text { H: tásçca, petorôso } \\
\text { T: petoròšo }\end{array}$ \\
\hline 88 & PRAĆANA & H: tâščica, petorôšo \\
\hline & FORŠIĆI & H: tâščica \\
\hline
\end{tabular}




$\begin{array}{ll}90 & \text { VRH } \\ 91 & \text { KRUŠVARI } \\ 92 & \text { LIPOGLAV } \\ 93 & \text { DRAGUĆ } \\ 94 & \text { BORUT } \\ 95 & \text { MAVRI } \\ 96 & \text { CEROVLJE } \\ 97 & \text { KATUN } \\ 98 & \text { BERAM } \\ 99 & \text { VELATRABA } \\ 100 & \text { LINDAR } \\ 101 & \text { PIĆAN } \\ & \\ 102 & \text { ČEPIĆ } \\ 103 & \text { SVETI PETAR U ŠUMI } \\ 104 & \text { PARIŽI } \\ 105 & \text { KATUNI } \\ 106 & \text { ŠUMBER } \\ 107 & \text { BLAŠKOVIĆI } \\ 108 & \text { ŽMINJ } \\ & \\ 109 & \text { PLOMIN } \\ & \\ 110 & \text { STRMAC } \\ 111 & \text { VELIGOLI } \\ 112 & \text { BREG } \\ 113 & \text { TRGET } \\ 114 & \text { SKITAČA } \\ 115 & \text { BROVINJE } \\ 116 & \text { VELE MUNE } \\ 117 & \text { MALE MUNE } \\ 118 & \text { ŽEJANE } \\ 119 & \text { MALI BRGUD } \\ 120 & \text { BRUS } \\ 121 & \text { ŠUŠNJEVICA } \\ 122 & \text { KOSTRČAN } \\ 123 & \text { NOVA VAS } \\ 124 & \text { JESENOVIK } \\ 125 & \text { IČIĆI } \\ 126 & \text { BRSEČ } \\ 127 & \text { OMIŠALJ } \\ 128 & \text { DOBRINJ } \\ 129 & \text { SVETI IVAN } \\ 130 & \text { BRUŠIĆ } \\ 131 & \text { SKRBČICI } \\ 132 & \text { KORNIĆ } \\ 133 & \text { KRK } \\ & \end{array}$

$\mathrm{H}$ : tầstica

H: tâščcica

H: tâščica

T: petiròšo, tàščica

H: tàščica

H: tâščica

H: tàščica, peterôšo

$\mathrm{H}$ : tâščica

H: tâščica

H: tâščica

H: petirôšo, tâščica

$\mathrm{T}$ : petoròšo

H: tâščica, krvãvčić

$\mathrm{H}$ : tâščica

$\mathrm{H}$ : tâščica

$\mathrm{H}$ : tâščica

$\mathrm{H}$ : tâščica

H: tâščica

H: tâščica

T: petoròšo

H: petorôšo

$\mathrm{T}$ : petoròšo

$\mathrm{H}$ : petirôšo

H: petorôšo

H: tâščica

$\mathrm{H}$ : tâščica

H: smrêkunčić, tâš̌ča, tăščica

H: tâščica

H: tâščica

H: kồtlarić

$\mathrm{H}$ : kồtlarić

R: puntaruôšu, puljî́ću de nè

H: tâščica

H: tâščica

R: tâška, tâškica

R: tâška čamâre

R: tuâškica

R: tâšsica

H: tàščica

H: tâščica

H: crvendầć

$\mathrm{H}$ : crvendâć

H: petarôš

H: crvendầć

H: babarûšica

H: crvendấć

$\mathrm{T}$ : peteròšo 


$\begin{array}{lll} & & \text { H: crvendầć } \\ 134 & \text { PUNAT } & \text { H: crvendầć } \\ 135 & \text { BAŠKA } & \text { H: pitarôšica }\end{array}$

ABBREVIAZIONI DELLE OPERE CITATE:

AIS Sprach - und Sachatlas Italiens und der Südschweitz, hgg. K. Jaberg und J. Jud, Zofingen 1928-40

ALI Atlante Linguistico Italiano, inedito; materiale consultabile presso l'Università di Torino

ANDR Jacques André, Les noms d'oiseaux en latin, Librairie C. Klincksieck, Paris, 1967

ASLEF Atlante storico - linguistico-etnografico friulano, I-IV, G.B. Pellegrini, Padova - Udine, 1972-1986

BEZLAJ Francè Bezlaj, Etimološki slovar slovenskega jezika, I-II (A-O), SAZU, Ljubljana, 1977, 1982

DLRM Dictionarul limbii romîne moderne, Academia republicii populare romîne, București, 1958

GREGORI J. Gregori - I. Krečič, Naši ptiči, DZS, Ljubljana, 1979

HIRTZ Miroslav Hirtz, Rječnik narodnih zoologičkih naziva, JAZU, Zagreb, 1938-1947

JaFa Vojmir Vinja, Jadranska fauna , I-II, JAZU, Zagreb, 1986

PETERSON R. Peterson - G. Mountfort - P.A.D. Hollom, Guida degli Uccelli d'Europa, Franco Muzzio and c. Editore, Padova, 1983

REW W. Meyer - Lübke, Romanisches Etymologisches Wörterbuch, Heilderberg, 1972

SKOK Petar Skok, Etimologijski rječnik hrvatskog ili srpskog jezika, I-IV, JAZU, Zagreb, 1972-1974

Prispevek obravnava imena za taščico (ERITHACUS RUBECULA) v vseh govorih v Istri (istriotsko, istrobeneško, istroromunsko, hrvaško, slovensko). Predstavi nam etimologije različnih poimenovanj za to ptico. Izrazi so bili zapisani $v 135$ istrskih krajih. 